\title{
PREDICTORS OF PSYCHIATRIC READMISSIONS IN THE SHORT- AND LONG-TERM: A POPULATION-BASED STUDY IN TAIWAN
}

\author{
Chuan-Hsiung Lin, ${ }^{\mathrm{I}}$ Wen-Ling Chen, ${ }^{\mathrm{II}}$ Chih-Ming Lin, ${ }^{\mathrm{III}}$ Ming-Der Lee RN, ${ }^{\mathrm{IV}}$ Ming-Chung Ko, ${ }^{\mathrm{V}}$ Chung-Yi Li ${ }^{\mathrm{II}}$
}

doi: 10.1590/S1807-59322010000500005

Lin CH, Chen WL, Lin CM, Lee MD, Ko MC, Li YC. Predictors of psychiatric readmissions in the short- and long-term: a population-based study in Taiwan. Clinics. 2010;65(5):481-9.

OBJECTIVES: To explore the risks and rates of readmission and their predictors 14 days, one year, and five years after discharge for the psychiatric population in Taiwan.

METHODS: This was a prospective study based on claims from 44,237 first-time hospitalized psychiatric patients discharged in 2000, who were followed for up to five years after discharge. The cumulative incidence and incidence density of readmission were calculated for various follow-up periods after discharge, and Cox proportional hazard models were generated to identify the significant predictors for psychiatric readmission.

RESULTS: The less than 14-day, one-year, and five-year cumulative incidences were estimated at $6.1 \%, 22.3 \%$, and $37.8 \%$, respectively. The corresponding figures for incidence density were 4.58, 1.04, and 0.69 per 1,000 person-days, respectively. Certain factors were significantly associated with increased risk of readmission irrespective of the length of follow-up, including male gender, length of hospital stay $>15$ days, economic poverty, a leading discharge diagnosis of schizophrenia/affective disorders, and residence in less-urbanized regions. Compared to children/adolescents, young adults (20-39 years) were significantly associated with increased risks of <one-year and < five-year readmissions, but not $<14$-day readmission. Additionally, hospital characteristics were significantly associated with increased risk of $<14$-day and $<$ one-year readmission, but not with risk of $<$ five-year readmission. CONCLUSIONS: This study found that the significant predictors for psychiatric readmission 14 days to five years after discharge were essentially the same except for patient's age and hospital accreditation level. This study also highlighted the importance of socioeconomic factors in the prediction of readmission.

KEYWORDS: Affective disorder; Health services research; Readmission; Schizophrenia; Social factor.

\section{INTRODUCTION}

The prevalence of psychiatric disorders has been increasing gradually in Taiwan since the implementation of Taiwan's National Health Insurance (NHI) Program in 1995. ${ }^{1,2}$ This could be because the universal health insurance

\footnotetext{
${ }^{\text {I }}$ Department of Health Care Administration, Central Taiwan University of Science and Technology - Taichung, Taiwan.

II Department of Health Care Management, National Taipei College of Nursing - Taipei, Taiwan.

III Department of Healthcare Information and Management, Ming Chuan University - Taoyuan County, Taiwan.

${ }^{\text {IV }}$ Graduate Institute of Long-Term Care, National Taipei College of Nursing

- Taipei, Taiwan.

${ }^{v}$ Department of Surgery, Taipei City Hospital - Taipei, Taiwan.

Email: a121752877@yahoo.com.tw

Received for publication on December 29, 2009

First review completed on January 27, 2010

Accepted for publication February 17, 2010
}

policy removed barriers to health care for the newly insured, enabling them more access to health care. ${ }^{1,2}$ Although the reported prevalence rate (under 1\%) of psychiatric disorders in Taiwan is much lower than that in Western countries, local studies concluded that psychiatric disorders are underdetected in Taiwan. ${ }^{3}$ Poorly treated psychiatric patients might be vulnerable to increased risks of extended hospital stays and rehospitalization, which may ultimately cause surplus medical expenditure. High readmission rate has been frequently considered an indicator of adverse prognostic outcomes in psychiatric inpatients. ${ }^{4}$ More importantly, a high readmission rate can reflect a large number of patients who cannot be satisfactorily accommodated in communities and can be used as an indicator of inadequacy or inappropriateness of community-based aftercare. ${ }^{5}$

Reported psychiatric readmission rates in the literature range from $10 \%$ for a one-month period of observation 
after discharge to as high as $86 \%$ within a sevenyear interval. ${ }^{6-10}$ Additionally, increased likelihood of readmission was observed to be associated with various factors, including predisposing factors such as prior history of psychiatric hospitalization, ${ }^{11-13}$ disease severity, alcohol and substance use, ${ }^{14}$ and lower level of patient function at discharge,,$^{15}$ as well as aftercare-related factors such as inadequate community support and ambulatory care visits. ${ }^{16}$ In general, readmission occurring very close to the time of discharge has been deemed as a valuable indicators strongly related to the quality of care delivered in hospitals. ${ }^{17}$ On the other hand, readmission that occurs at a time distant from hospital discharge can be influenced not only by quality of care but also by aftercare and psychiatric services provided in both community and outpatient settings. Thus, comparisons between readmission rates close to the time of discharge and those distant from discharge may provide a better understanding of the quality of aftercare and other family or community support received by psychiatric patients.

In addition to information concerning the difference between readmission rates close to and distant from discharge, there is also a need to acknowledge the respective predictors for short-term and long-term risks of readmission. Such information could allow the identification of patients at immediate risk of readmission after discharge and those still at greater risk of readmission during a longer period of time after discharge. Different care strategies should be considered and offered to patients with immediate risk of readmission and to those at greater risk over a longer time period after discharge. The readmission rate and its correlates might vary with both the study population and length of follow-up, and there have been no studies conducted using the same study cohort to compare between readmission rates close to and distant from discharge. We thus conducted a five-year population-based cohort study to obtain reliable estimates of $<14$-day, <one-year, and <fiveyear readmission rates and their significant predictors in first-time hospitalized psychiatric patients. We hypothesized that patient characteristics may have differential influences on readmission close to and distant from discharge, and that characteristics related to hospital and ward may have greater influence on <14-day readmission than on <one-year and $<$ five-year readmission.

\section{METHODS}

\section{Source of data and study design}

Data analyzed in this study were retrieved from three claim files of the National Health Insurance Research
Database (NHIRD) provided by the Bureau of National Health Insurance (BNHI). These datasets included the Inpatient Expenditures by Admissions (IEA), Registry for Contracted Medical Facilities (RCMF), and Registry for Beneficiaries (RB). The NHIRD provides all inpatient and ambulatory medical claims for around $96 \%$ of the Taiwanese people. ${ }^{2}$ To ensure the accuracy of claim files, the BNHI performs an expert review on a random sample of every 50 to 100 ambulatory and inpatient claims quarterly, and false diagnoses face severe penalties. ${ }^{18}$ By the end of 1996, BNHI had contracted with $97 \%$ of hospitals and clinics all over the nation, and $99 \%$ of the total Taiwanese population had already enrolled in the program. ${ }^{2}$ Access to research data was approved by the Review Committee of the National Health Research Institutes.

This was a cohort study that evaluated the period between 2000 and 2005. Data from the 2000 IEA showed a total of 114,330 episodes of hospitalization due to psychiatric causes (International Classification of Disease, the $9^{\text {th }}$ version with Clinical Modification (ICD9-CM) code: 290-319) in Taiwan. These hospitalizations comprised 62,290 patients. For those who had more than one episode of hospitalization in 2000, we kept the firsttime episode in 2000 as the index hospitalization. Each record of IEA provides various pieces of information, including a personal identification number (PIN), date of birth, sex, and dates of admission and discharge with one primary code and up to four secondary diagnostic codes. We linked, through their PIN, the patients hospitalized in 2000 to the IEA to evaluate possible prior hospitalization in the years of 1997-1999 and found that there were 17,357 patients who had a history of psychiatric hospitalization between 1997 and 1999. These patients were then excluded. We further excluded 696 patients who were deceased during hospital stay, transferred to other hospitals, or discharged against medical advice, resulting in 44,237 hospitalized patients who were considered as first-time hospitalized patients and as the subjects of the study cohort.

The study cohort $(\mathrm{N}=44,237)$ was then linked to IEA for the years 2000-2005 to identify possible readmission episodes after discharge. After excluding the planned readmissions recorded in the claim files, 16,718 (37.8\%) patients were readmitted within five years of discharge. Of those readmitted, 2,678 (6.1\%) and 9,881 (22.3\%) were readmitted within 14 days and one year after discharge, respectively. We also linked the study cohort to the 2000 RCMF to obtain the hospital information of the initial (index) hospitalization and to the 2000-2004 RB for patient information on insurance premiums and dates of possible termination of health insurance policies. 


\section{Potential predictors of readmission}

The potential predictors of readmission explored in this study included characteristics of patients and hospitals. The age of a patient was calculated as the time period in years between the date of birth and date of discharge for the index hospitalization. We used the paid insurance premiums as a surrogate for patient socioeconomic backgrounds, which were categorized into 0 (non-employed) and tertile ranges, i.e., 1-1,007 (poorer), 1,008-19,200, and $>=19,201$ (richer) New Taiwan Dollars (NTD). We also examined the influences of common psychiatric diagnoses including schizophrenic disorders (ICD-9-CM code: 295) and affective psychoses (ICD-9-CM code: 296) on the risk of readmission; these appeared as the primary discharge diagnoses for the index hospitalizations. The length of stay (LOS) was calculated as the time period between the date of hospitalization and date of discharge, and was categorized according to a quartile distribution into four groups, i.e., $0-4$, 5-7, 8-15, and $>15$ days.

All hospitals in Taiwan are required to be evaluated and accredited primarily based on their service volumes, range of specialties, and a comprehensive list of quality of carerelated indicators. Medical centers are the hospitals with the best overall evaluation results, followed by regional hospitals and local hospitals. A hospital's location reflects the level of urbanization of the local area. An adjustment for urbanization was performed to remove the potential influences of differential criteria adopted by hospitals for readmission, as well as differential accessibility of patients living in different areas.

\section{Statistical analysis}

Readmission was considered as the primary outcome variable in this study and was calculated for three different time periods after discharge, i.e., <14 days, <one year, and $<$ five years. We first calculated the overall cumulative incidence (CuI) and incidence density (ID) of readmission and then calculated CuIs according to the characteristics of patients and hospitals. Those readmitted during a shorter time period were also included in calculations of readmissions for longer periods of time. The significant predictors for readmission were explored with multivariate Cox proportional hazard models. In constructing Cox models, the follow-up began on the date of discharge. The study subjects who withdrew (including those who died) from the NHI program were censored, and the date of censoring was the date on which they terminated the insurance policy. If the patient did not leave the NHI program and encountered no readmission, the date of censoring was the date of the end of follow-up (i.e., the last day of the different follow-up periods). We validated the Cox regression model by checking whether the assumption of proportionality held. The $\log (-\log )$ graph for each independent variable indicated no deviation from the assumption of proportional hazards. Thus, the Cox regression model could effectively and accurately identify the psychiatric patients at greater risk of readmission. We also estimated, using the life-table method, ${ }^{19}$ readmission rates and cumulative survival rates for the study cohort. SPSS (Chicago, IL; ver. 12.0) software was used for the statistical analyses. $\mathrm{p}<0.05$ was considered statistically significant.

\section{RESULTS}

The overall CuIs within 14 days, one year, and five years of discharge were estimated at $6.1 \%, 22.3 \%$, and $37.9 \%$, respectively. The corresponding figures for ID were $4.58,1.04$, and 0.69 per 1,000 person-days, respectively. Male patients had a higher $\mathrm{CuI}$ of readmission than female patients regardless of the length of follow-up. Compared to the overall $\mathrm{CuI}$, patients aged $<40$ years had a higher $\mathrm{CuI}$ of readmission in 14 days $(6.5 \%-8.6 \%)$ and in one year (20.8\%-29.9\%), and those aged 20-49 had a higher <fiveyear CuI (38.0\%-48.5\%). The patients with an LOS of $>15$ days had the greatest $\mathrm{CuI}$ for various lengths of follow-up (<14-day, 9.9\%; <one-year, 34.0\%; and <five-year, $54.4 \%$ ) compared to the other patients (<14-day, $2.9 \%-5.7 \%$; <oneyear, $16.1 \%-22.9 \%$; <five-year, $28.7 \%-38.3 \%$ ). Additionally, poorer patients $(8.7 \%)$ and those who had a primary discharge diagnosis of schizophrenia (13.6\%) or affective disorder $(8.7 \%)$ also had higher CuIs in 14-days. The <oneyear and $<$ five-year CuIs for the poorer patients and patients with schizophrenia or affective disorders remained higher. There was an apparent variation in CuIs of readmission across geographic locations and accreditation levels of hospitals. Patients discharged from hospitals located in the Kao-Ping and Eastern districts (less urbanized areas) or from district hospitals not only had higher $<14$-day CuIs $(7.2 \%$, $8.4 \%$, and $6.7 \%$, respectively) but also had higher <one-year $(24.1 \%, 26.7 \%$, and $23.7 \%$, respectively) and $<$ five-year $\mathrm{CuI}$ $\mathrm{s}(39.2 \%, 45.2 \%$, and $38.9 \%$, respectively) (Table 1$)$.

Table 2 shows adjusted hazard ratios (AHRs) of readmission for various follow-up periods in association with various characteristics of patients and hospitals. Female patients were significantly associated with reduced risks of $<14$-day $($ AHR $=0.88,95 \%$ confidence interval $(\mathrm{CI})=0.81$ $0.95)$, <one-year (AHR $=0.85,95 \% \mathrm{CI}=0.82-0.89)$ and $<$ five-year readmission (AHR $=0.90,95 \% \mathrm{CI}=0.87-0.92$ ). Compared to adolescent and child patients, adult patients all 
Table 1 - Readmission rates within various time periods after discharge according to the characteristics of patients and hospitals $(n=44,237)$.

\begin{tabular}{|c|c|c|c|c|}
\hline \multirow{2}{*}{ Variables } & \multirow{2}{*}{$\mathrm{N}$} & \multicolumn{3}{|c|}{ Readmission rate $(\%)$} \\
\hline & & $<14$ days $(\mathrm{N}=2,678)$ & $<1$ year $(\mathrm{N}=9,881)$ & $<5$ years $(\mathrm{N}=16,752)$ \\
\hline \multicolumn{5}{|c|}{ Patient's characteristics } \\
\hline \multicolumn{5}{|l|}{ Gender } \\
\hline Male & 23,778 & 6.5 & 24.0 & 39.7 \\
\hline Female & 20,459 & 5.5 & 20.4 & 36.0 \\
\hline \multicolumn{5}{|l|}{ Age (years) } \\
\hline$<20$ & 2,230 & 6.5 & 20.8 & 33.4 \\
\hline $20-29$ & 4,828 & 8.6 & 29.9 & 48.5 \\
\hline $30-39$ & 7,351 & 7.2 & 27.4 & 46.3 \\
\hline $40-49$ & 7,165 & 5.5 & 21.4 & 38.0 \\
\hline $50-59$ & 4,906 & 5.1 & 19.2 & 33.3 \\
\hline $60-69$ & 5,584 & 4.4 & 16.7 & 30.6 \\
\hline$>=70$ & 12,173 & 5.7 & 21.0 & 34.5 \\
\hline Mean \pm SD & $52.1 \pm 21.1$ & & & \\
\hline \multicolumn{5}{|c|}{ Length-of-hospital stay (days) } \\
\hline $0-4$ & 14,726 & 4.9 & 16.1 & 28.7 \\
\hline $5-7$ & 8,992 & 3.9 & 18.0 & 32.9 \\
\hline $8-15$ & 9,824 & 5.7 & 22.9 & 38.3 \\
\hline$>15$ & 10,695 & 9.9 & 34.0 & 54.4 \\
\hline Mean \pm SD & $14.6 \pm 23.0$ & & & \\
\hline \multicolumn{5}{|c|}{ Insurance premium $\left(\mathrm{NTD}^{\mathrm{a}}\right)$} \\
\hline 0 & 12,539 & 5.8 & 21.3 & 35.9 \\
\hline $1-1,007$ & 11,387 & 8.7 & 28.7 & 46.2 \\
\hline $1,008-19,200$ & 15,250 & 4.9 & 20.0 & 35.3 \\
\hline$>=19,201$ & 5,061 & 4.2 & 17.4 & 32.0 \\
\hline Mean \pm SD & $10,361 \pm 12,319$ & & & \\
\hline \multicolumn{5}{|c|}{ Leading discharge diagnosis } \\
\hline Others & 34,426 & 4.5 & 18.0 & 30.9 \\
\hline Schizophrenia & 5,923 & 13.6 & 41.0 & 67.2 \\
\hline Affective disorder & 3,888 & 8.7 & 32.7 & 55.1 \\
\hline \multicolumn{5}{|c|}{ Hospital characteristics } \\
\hline \multicolumn{5}{|l|}{ Location } \\
\hline Taipei & 13,324 & 5.3 & 22.5 & 39.3 \\
\hline North & 6,473 & 5.9 & 21.6 & 35.7 \\
\hline Central & 8,695 & 5.6 & 20.5 & 35.5 \\
\hline South & 5,488 & 6.1 & 21.5 & 36.2 \\
\hline Kao-Ping & 8,438 & 7.2 & 24.1 & 39.2 \\
\hline East & 1,819 & 8.4 & 26.7 & 45.2 \\
\hline \multicolumn{5}{|l|}{ Accreditation level } \\
\hline Medical center & 10,371 & 5.4 & 21.5 & 38.4 \\
\hline Regional hospital & 14,606 & 5.6 & 21.2 & 36.2 \\
\hline District hospital & 19,260 & 6.7 & 23.7 & 38.9 \\
\hline Total & 44,237 & 6.1 & 22.3 & 37.9 \\
\hline
\end{tabular}

${ }^{a}$ NTD=New Taiwan Dollar (1 USD(32 NTD). 
Table 2 - Adjusted hazard ratios of $<14$-day and $<$ five-year readmission rates in association with various characteristics of patients and hospitals $(n=44,237)$.

\begin{tabular}{|c|c|c|c|c|c|c|}
\hline \multirow[t]{2}{*}{ Variables } & \multicolumn{2}{|c|}{$<14$-day readmission } & \multicolumn{2}{|c|}{$<1$-year readmission } & \multicolumn{2}{|c|}{$<5$-year readmission } \\
\hline & $\operatorname{AHR}^{\mathrm{a}}(95 \% \mathrm{CI})$ & $\mathrm{p}$ & $\operatorname{AHR}^{a}(95 \% \mathrm{CI})$ & $\mathrm{p}$ & AHR $(95 \% \mathrm{CI})$ & $\mathrm{p}$ \\
\hline \multicolumn{7}{|l|}{ Patient characteristics } \\
\hline \multicolumn{7}{|l|}{ Gender } \\
\hline Male & 1.00 & & 1.00 & & 1.00 & \\
\hline Female & $0.88(0.81-0.95)$ & 0.002 & $0.85(0.82-0.89)$ & $<0.001$ & $0.90(0.87-0.92)$ & $<0.001$ \\
\hline \multicolumn{7}{|l|}{ Age (years) } \\
\hline$<20$ & 1.00 & & 1.00 & & 1.00 & \\
\hline $20-29$ & $0.97(0.80-1.19)$ & 0.796 & $1.19(1.07-1.33)$ & 0.002 & $1.28(1.17-1.39)$ & $<0.001$ \\
\hline $30-39$ & $0.91(0.75-1.11)$ & 0.337 & $1.15(1.04-1.28)$ & 0.009 & $1.26(1.16-1.37)$ & $<0.001$ \\
\hline $40-49$ & $0.79(0.64-0.97)$ & 0.021 & $0.98(0.88-1.10)$ & 0.741 & $1.10(1.01-1.19)$ & 0.037 \\
\hline $50-59$ & $0.84(0.68-1.04)$ & 0.101 & $0.95(0.85-1.07)$ & 0.435 & $1.02(0.93-1.12)$ & 0.658 \\
\hline $60-69$ & $0.76(0.62-0.94)$ & 0.012 & $0.83(0.74-0.93)$ & 0.001 & $0.94(0.86-1.03)$ & 0.163 \\
\hline$>=70$ & $0.99(0.83-1.21)$ & 0.968 & $1.06(0.95-1.17)$ & 0.295 & $1.10(1.02-1.20)$ & 0.018 \\
\hline \multicolumn{7}{|c|}{ Length of hospital stay (days) } \\
\hline $0-4$ & 1.00 & & 1.00 & & 1.00 & \\
\hline $5-7$ & $0.79(0.70-0.90)$ & $<0.001$ & $1.11(1.04-1.18)$ & 0.002 & $1.15(1.10-1.21)$ & $<0.001$ \\
\hline $8-15$ & $1.07(0.95-1.20)$ & 0.276 & $1.37(1.29-1.46)$ & $<0.001$ & $1.33(1.27-1.39)$ & $<0.001$ \\
\hline$>15$ & $1.37(1.23-1.53)$ & $<0.001$ & $1.67(1.57-1.77)$ & $<0.001$ & $1.57(1.50-1.65)$ & $<0.001$ \\
\hline \multicolumn{7}{|c|}{ Insurance premium $\left(\mathrm{NTD}^{\mathrm{a}}\right)$} \\
\hline 0 & 1.00 & & 1.00 & & 1.00 & \\
\hline $1-1,007$ & $1.25(1.13-1.39)$ & $<0.001$ & $1.12(1.06-1.19)$ & $<0.001$ & $1.12(1.07-1.17)$ & $<0.001$ \\
\hline $1,008-19,200$ & $0.83(0.74-0.93)$ & 0.001 & $0.89(0.84-0.94)$ & $<0.001$ & $0.93(0.90-0.97)$ & 0.002 \\
\hline$>=19,201$ & $0.80(0.68-0.95)$ & 0.009 & $0.77(0.71-0.84)$ & $<0.001$ & $0.81(0.76-0.86)$ & $<0.001$ \\
\hline \multicolumn{7}{|c|}{ Leading discharge diagnosis } \\
\hline Others & 1.00 & & 1.00 & & 1.00 & \\
\hline Schizophrenia & $2.63(2.33-2.86)$ & $<0.001$ & $1.96(1.85-2.08)$ & 0.005 & $2.17(2.04-2.27)$ & $<0.001$ \\
\hline Affective disorder & $1.95(1.51-2.40)$ & $<0.001$ & $1.76(1.56-2.06)$ & $<0.001$ & $1.89(1.67-2.09)$ & $<0.001$ \\
\hline \multicolumn{7}{|c|}{ Hospital characteristics } \\
\hline \multicolumn{7}{|l|}{ Location } \\
\hline Taipei & 1.00 & & 1.00 & & 1.00 & \\
\hline North & $1.23(1.08-1.40)$ & 0.002 & $1.07(1.00-1.14)$ & 0.058 & $1.01(0.96-1.06)$ & 0.713 \\
\hline Central & $1.17(1.03-1.32)$ & 0.013 & $1.03(0.97-1.09)$ & 0.387 & $1.01(0.96-1.06)$ & 0.664 \\
\hline South & $1.19(1.04-1.36)$ & 0.012 & $1.03(0.96-1.10)$ & 0.452 & $1.00(0.95-1.06)$ & 0.978 \\
\hline Kao-Ping & $1.46(1.31-1.64)$ & $<0.001$ & $1.16(1.10-1.23)$ & $<0.001$ & $1.08(1.03-1.13)$ & 0.001 \\
\hline East & $1.36(1.14-1.63)$ & 0.001 & $1.13(1.02-1.25)$ & 0.015 & $1.14(1.05-1.22)$ & 0.001 \\
\hline \multicolumn{7}{|l|}{ Accreditation level } \\
\hline Medical center & 1.00 & & 1.00 & & 1.00 & \\
\hline Regional hospital & $1.21(1.08-1.35)$ & 0.001 & $1.11(1.05-1.18)$ & $<0.001$ & $1.04(1.00-1.09)$ & 0.058 \\
\hline District hospital & $1.29(1.14-1.46)$ & $<0.001$ & $1.18(1.11-1.26)$ & $<0.001$ & $1.06(0.98-1.15)$ & 0.224 \\
\hline
\end{tabular}

a AHR=adjusted hazard ratio; $\mathrm{CI}=$ confidence interval; NTD=New Taiwan Dollar (1 USD $\approx 32$ NTD).

had lower AHRs of <14-day readmission, ranging from 0.76 to 0.99 , but only the reduced AHRs for patients aged 40-49 $(0.79,95 \% \mathrm{CI}=0.64-0 / 97)$ and $60-69(0.76,95 \% \mathrm{CI}=0.62-$ $0.94)$ were statistically significant. Young adults aged $<40$ years, however, experienced increased risk of readmission as the length of follow-up extended to one year (20-29 years:
AHR $=1.19,95 \% \mathrm{CI}=1.07-1.33 ; 30-39$ years: $\mathrm{AHR}=1.15$, 95\% CI=1.04-1.28). The AHR of $<$ five-year readmission for those young adults further increased to $1.28(95 \% \mathrm{CI}=1.17$ 1.39 ) and 1.26 (95\% CI=1.16-1.37), respectively. LOS also had differential influences on readmission estimated for various lengths of follow-up. Compared to an LOS of 0-4 
days, an LOS of 5-7 days was associated with a significantly reduced risk of $<14$-day readmission $(\mathrm{AHR}=0.79,95 \%$ $\mathrm{CI}=0.70-0.90)$ and with a significantly increased risk of <one-year (AHR=1.11, 95\% CI=1.04-1.18) and <five-year readmission $(\mathrm{AHR}=1.15,95 \% \mathrm{CI}=1.10-1.21)$. On the other hand, a longer LOS $(>15)$ was significantly associated with an elevated AHR in a range between 1.37 (95\% CI=1.231.53 ; <14-day readmission) and 1.67 (95\% CI=1.57-1.77; $<$ one-year readmission). Compared to non-employed patients, poorer patients consistently had increased risk of readmission within 14 days $(\mathrm{AHR}=1.25,95 \% \mathrm{CI}=1.13$ 1.39), one year $(\mathrm{AHR}=1.12,95 \% \mathrm{CI}=1.06-1.19)$, and five years after discharge $(\mathrm{AHR}=1.12,95 \% \mathrm{CI}=1.07-1.17)$. On the other hand, those with higher insurance premiums consistently had significantly reduced AHRs irrespective of the length of follow-up. Schizophrenic patients $(\mathrm{AHR}=2.63$, 95\% CI=2.33-2.86) and those with affective psychoses (AHR $=1.95,95 \% \mathrm{CI}=1.51-2.40)$ both had a significantly increased risk of $<14$-day readmission. These two common psychiatric diagnoses were also significantly, but to a lesser extent, associated with an increased AHR of <one-year and $<$ five-year readmissions.

Compared to the patients treated in Taipei city, the nation's capital, those discharged from hospitals in less-urbanized areas were more likely to be readmitted within 14 days after discharge, with AHRs ranging from 1.17 (95\% CI=1.03-1.32) in the Central area to 1.46 (95\% CI=1.31-1.64) in the KaoPing area. However, only the Kao-Ping and Eastern districts (the least urbanized areas) were significantly associated with an increased risk of <one-year and $<$ five-year readmissions. Additionally, patients discharged from hospitals with lower accreditation levels had a significantly increased risk of $<14$ day readmission [regional hospital: AHR=1.21 (95\% CI=1.081.35); district hospital: $\mathrm{AHR}=1.29$ (95\% CI=1.14-1.46)] and <one-year readmission [regional hospital: AHR=1.11 (95\% $\mathrm{CI}=1.05-1.18)$; district hospital: $\mathrm{AHR}=1.18$ (95\% CI=1.11$1.26)$ ], but not $<$ five-year readmission.

Table 3 shows the rate of readmission over the five-year observational period. The highest readmission rate was noted at the end of the first year after discharge ( 0.23 per person-day); the corresponding figures for the other years were smaller, ranging from 0.05 to 0.09 per person-day. The cumulative survival rate was estimated to be 0.57 by the end of the fifth year after discharge (Figure 1).

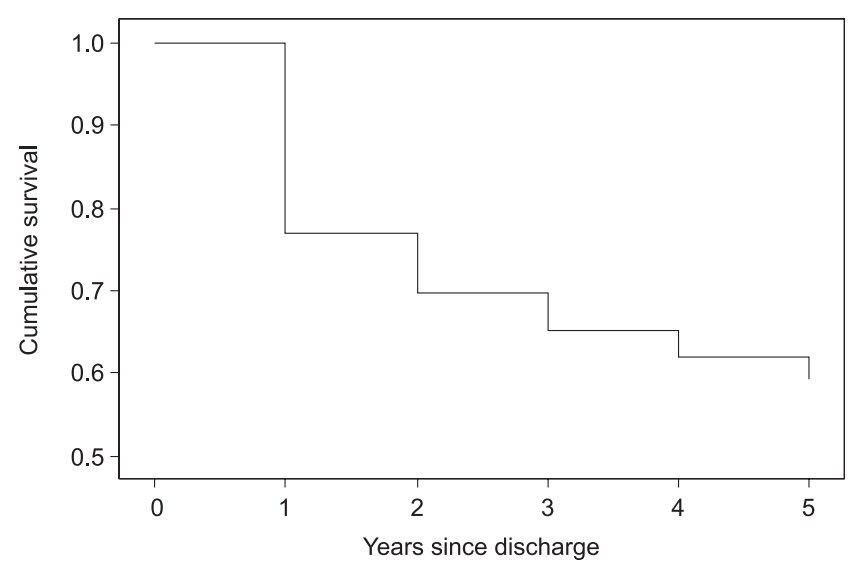

Figure 1 - Cumulative survival rate for the study cohort within five years after discharge.

\section{DISCUSSION}

\section{Main findings}

Our findings show that $6.1 \%$ of psychiatric patients were readmitted within 14 days after discharge, and the number increased to $37.8 \%$ as the observational period extended to five years. At the end of the fifth year, the cumulative survival rate was estimated to be 0.57 . Additionally, a LOS of $>15$ days, male gender, schizophrenia/affective disorder, and poorer socioeconomic status were all significant predictors of elevated risk of readmission, irrespective of the length of follow-up. We also found that age and a LOS of 5-7 days had different influences on <14-day readmission and on readmissions estimated for one-year and five-year follow-up periods. The hospital accreditation level had a greater effect on the risk of <14-day readmission than on the readmission risk over longer periods of time, which is consistent with our research hypothesis.

\section{Predictors of readmission}

An earlier Swiss study noted that female psychiatric patients were more vulnerable than male patients to

Table 3 - Rate of readmission and survival during five years after discharge.

\begin{tabular}{cccccc}
\hline Year since discharge & Person-days at risk & No. of readmissions & Readmission rate & Survival rate & Cumulative survival rate \\
\hline 0 & $42,821.0$ & 9,864 & 0.23 & 0.77 & 0.91 \\
0.77 & 0.70 \\
1 & $31,023.5$ & 2,896 & 0.09 & 0.94 & 0.65 \\
3 & $27,255.5$ & 1,766 & 0.05 & 0.95 & 0.62 \\
4 & $24,801.5$ & 1,269 & 0.08 & 0.92 & 0.57 \\
\hline
\end{tabular}


experience multiple readmissions within one year after discharge..$^{20}$ Two recent studies conducted in the $\mathrm{US}^{21}$ and Singapore, ${ }^{22}$ on the other hand, found that male gender was associated with an increased risk of 20-month and one-year readmission rates among acute psychiatric inpatients and schizophrenic patients, respectively. Our study showed that male gender was a significant risk factor not only for $<14$-day readmission but also for $<$ five-year readmission. Alcohol and substance use have been frequently found to be risk factors for psychiatric readmission, ${ }^{10,13,14}$ and these adverse habits are believed to be more prevalent in males than in females. Whether the gender differences in both $<14$-day and $<$ fiveyear readmissions reflect a higher prevalence of alcohol and substance use in male patients warrants further investigations.

We found that adult patients had a lower risk of $<14$-day readmission but tended to have a greater risk of <one-year and $<$ five-year readmission. This finding provides support to our research hypothesis that individual characteristics may have differential influences on readmission occurring at times close to and remote from discharge. A Norwegian study reported that younger age may predict an increased readmission rate in a one-year period after discharge. ${ }^{23} \mathrm{~A}$ US study also investigated the three-month readmission rate in both children and adolescents and indicated that children with learning disabilities and delayed development were at an increased risk of readmission within a short period of time after discharge. ${ }^{24}$ These enabling factors could be responsible for the higher <14-day readmission rate in children and adolescents noted in our study. On the other hand, a number of social and behavioral factors, such as homelessness ${ }^{10}$, alcohol and substance abuse,,$^{10,13,14,20}$ and being single or living alone, ${ }^{21,25}$ were found to be related to readmission rates over a longer period of time (from one to seven years) after discharge. These social and behavioral enabling factors are believed to be more prevalent in adult patients than in children and adolescent patients, and might contribute to an increased risk of <one-year and <five-year readmissions in adults. Moreover, the frequency of posthospital ambulatory care and the receipt of community rehabilitation, which can effectively reduce the risk of readmission, are likely to be more adequate for children/ adolescents than for adult patients because most children/ adolescent patients are likely to receive attention from their healthy parents.

Our study found that schizophrenic and affective disorders may increase the risk of $<14$-day and <one-year readmission, which is consistent with the findings of some previous studies that investigated predictors of readmission within six months ${ }^{8}$ or one year ${ }^{26}$ after discharge. Our study further demonstrated that patients with these two disorders had an increased risk of readmission beyond the first year after discharge. These findings may highlight the importance of in-hospital care and post-hospital aftercare for schizophrenic and affective psychotic patients.

We found a curve-linear relationship between LOS and $<14$-day readmission, in which a LOS of five to seven days was associated with the most reduced risk of $<14$-day readmission. Such a curve-linear pattern was in accord with previous findings that inadequate LOS may result in a higher risk of admission, ${ }^{26}$ and patients with a longer hospital stay might simply be sicker, homeless or lacking adequate social support and living arrangements, ${ }^{15}$ which might all lead to a higher $<14$-day readmission rate. Interpretation of the relationship between LOS and the risks of readmission one year and five years after discharge might not be straightforward because Mortensen and Eaton reported that the relationship between LOS and the <five-year risk of readmission can be greatly affected by the availability of aftercare. ${ }^{27}$ Such mediating effects of aftercare or other socially related factors such as living arrangement and social support might also be responsible for the observed lack of association between the <five-year readmission rate and hospital accreditation, which had significant impact on $<14$ day readmission.

Comprehensive evidence has shown that social factors are strongly related to the readmission rate in psychiatric patients. These factors included homelessness, ${ }^{10}$ being single, ${ }^{21}$ living alone, ${ }^{23}$ unemployment, ${ }^{23}$ limited social support, ${ }^{15}$ and living in a rural region. ${ }^{11}$ The study by Kent et al. in Australia attempted to identify factors that contributed to psychiatric readmission and found that social factors contributed to $38.9 \%$ of readmissions, followed by factors related to psychiatric and physical illness $(31.1 \%)$, being dangerousness to self or others $(20.3 \%)$ and substance abuse $(9.7 \%) .{ }^{28}$ Although our study did not have a direct measure of patient socioeconomic status, some socioeconomic factors including insurance premiums and the location of the discharge hospital showed that poorer patients and those discharged from hospitals in the least urbanized regions were at greater risks of not only $<14$-day readmission but also <one-year and <five-year readmission, which again highlights the importance of social factors for the risk of psychiatric readmission. The contribution of social factors to the readmission of psychiatric patients is strong evidence that the mental health system should provide appropriate targeted resources and assertive, continuous care management to avoid social crises.

\section{Strengths and limitations}

Our study has several methodological strengths. First, unlike most previous studies that explored significant 
predictors for readmission rates within a fixed period of time after discharge, our study investigated the predictors of readmission within different periods of time after discharge using the same study cohort. This approach may have important healthcare policy implications because the predictors for readmission very close to discharge and those for readmission distant from discharge might be different, ${ }^{9,17}$ and the difference between readmission rates close to and remote from discharge can be used to assess the adequacy of aftercare for psychiatric patients. Second, the number of previous hospitalizations has consistently been found to be a risk factor for readmission. ${ }^{11-13,16,29,30}$ We limited our study sample to patients without a prior history of psychiatric hospitalization, which avoids the potential confounding effect of previous psychiatric hospitalization. Third, this study was based on a very large sample size, allowing us to explore independent effects of numerous potential predictors simultaneously. Fourth, this analysis was based on the national NHI dataset, which leaves little room for selection bias or attrition of study subjects due to loss to follow-up.

There were also several limitations in our study. First, the hospital admission rate can be affected by health-related policies. ${ }^{31}$ Thus, our study results might be affected by differing policies of admission adopted by different hospitals. Second, although we considered the adjusted primary diagnosis at discharge, age, sex, and socioeconomic status indicated by insurance premiums and areas of residence, a number of potential confounding factors including co-morbidities, disease severity, lifestyle, and behavior were not considered due to a lack of such information in the NHI claims. This could result in residual confounding effects by these variables when assessing the independent influence of the potential predictors explored in this study. Most importantly, several factors including familial living arrangement, social support, and marital status ${ }^{15,20}$ were found to be strongly related to psychiatric readmission rates, and these variables were not taken into account in the analysis. Failure to adjust for these factors might further limit the interpretation of the observed relationships between characteristics of patients and hospitals and risk of readmission. Finally, Taiwan's National Health Insurance program was implemented in 1994, and the earliest year when electronic claim files were available was 1997. Therefore, we were unable to obtain claim data for years earlier than 1997, which makes it very likely that an unknown proportion of participants had at least one previous psychiatric admission before 1997.

\section{Implications and conclusion}

This study found a psychiatric readmission rate of 4.58 per 1,000 person-days in the first 14 days after discharge, and the figures decreased substantially to 1.04 and 0.69 per 1,000 person-days as the length of follow-up extended to one year and five years. These numbers indicate a high rate of readmission in a very short period of time after discharge and suggest a need for further improvement in care strategies and discharge planning, especially for high-risk groups. Although the rate of readmission was high within 14 days after discharge, our study clearly demonstrated that many patients were also admitted in the five years following discharge, and this may suggest an inadequacy of aftercare strategies in Taiwan. Local clinicians and policy makers should explore specific forms of aftercare and types of services that can ensure that discharged psychiatric patients remain in the community. These aftercare strategies would be generally needed for young adult patients whose risk of readmission increased later in the follow-up period. Moreover, implementation of special care strategies or reallocation of medical and social resources should be seriously considered for poorer patients and those from the least urbanized regions in Taiwan. Better management of patients with schizophrenia or affective disorder who normally have longer hospital stays may also help to effectively reduce the likelihood of readmission during the five years after discharge.

In conclusion, we found that hospital characteristics were related more to $<14$-day readmission than to readmission rates over periods of one year or longer. Our study also highlights the importance of socioeconomic factors in the prediction of $<14$-day, <one-year, and <five-year readmission, in which poorer patients and those from the least urbanized regions were especially vulnerable to readmission. Early identification of the subset of psychiatric patients likely to be readmitted at a time close to or remote from discharge is essential for planning and implementing specific care strategies, either inhospital or post-hospital, to further reduce the readmission of psychiatric patients in Taiwan.

\section{REFERENCES}

1. Cheng SH, Chiang TL. The effect of universal health insurance on health care utilization in Taiwan. Results from a natural experiment. JAMA. 1997;278:89-93.
2. Lu JF, Hsiao WC. Does Universal health insurance make health care unaffordable? lessons from Taiwan. Health Aff. 2003;22:77-88. 
3. Chien IC. Prevalence, use, and costs of psychiatric disorders among National Health Insurance enrollees in Taiwan. Taipei: Institute of Public Health, National Yang-Ming University; Dissertation 2004.

4. Häfner H, van der Heiden W. The evaluation of mental health care systems. Br. J. Psychiatry. 1989;155:12-7.

5. Thornicroft G, Gooch C, Dayson D. Readmissions to hospital for long term psychiatric patients after discharge to the community. Br. Med. J.1992;305:996-8.

6. Lin $\mathrm{CH}$, Chen CC, Wang SY, Lin SC, Chen MC, Lin CH. Factors affecting time to rehospitalization in Han Chinese patients with schizophrenic disorder in Taiwan. Kaohsiung J Med Sci. 2008;24:40814.

7. Appleby L, Desai PN, Luchins DJ, Gibbons RD, Hedeker DR. Length of stay and recidivism in schizophrenia. Am J Psychiatry.1993;150:72-6.

8. Prince JD, Akincigil A, Kalay E, Walkup JT, Hoover DR, Lucas J, et al. Psychiatric rehospitalization among elderly persons in the United States. Psychiatr Serv. 2008; 59:1038-45.

9. Herceg M, Jukić V, Vidović D, Erdeljić V, Celić I, Kozumplik O, et al. Two-year rehospitalization rates of patients with newly diagnosed or chronic schizophrenia on atypical or typical antipsychotic drugs: retrospective cohort study. Croat Med J. 2008;49:215-23.

10. Irmiter C, McCarthy JF, Barry KL, Soliman S, Blow, FC. Reinstitutionalization following psychiatric discharge among VA patients with serious mental illness: a national longitudinal study. Psychiatr Q.2007;78:279-86

11. Roick C, Heider, D, Kilian R, Matschinger H, Toumi M, Angermeyer, MC. Factors contributing to frequent use of psychiatric inpatient services by schizophrenia patients. Soc. Psychiatry Psychiatr. Epidemiol.2004;39:744-51.

12. Olfson M, Mechanic D, Boyer CA, Hansell S, Walkup J, Weiden PJ . Assessing clinical predictions of early rehospitalization in schizophrenia. J Nerv Ment Dis. 1999; 187:721-9.

13. Lin $\mathrm{CH}$, Chen YS, Lin $\mathrm{CH}$, Lin KS. Factors affecting time to rehospitalization for patients with major depressive disorder. Psychiatry Clin. Neurosci. 2007;61:249-54.

14. Bimerew MS, Sonn FC, Kortenbout WP. Substance abuse and the risk of readmission of people with schizophrenia at Amanuel Psychiatric Hospital, Ethiopia. Curationis. 2007;30:74-81.

15. Mercer GT, Molinari V, Kunik ME, Orengo CA, Snow L, Rezabek P. Rehospitalization of older psychiatric inpatients: an investigation of predictors. Gerontologist.1999;39:591-8.

16. Walker R, Minor-Schork D, Bloch R, Esinhart J. High risk factors for rehospitalization within six months. Psychiatr Q.1996;67:235-43
17. Kramer MS, Demissie K, Yang H, Platt RW, Sauvé R, Liston R. The contribution of mild and moderate preterm birth to infant mortality. Fetal and Infant Health Study Group of the Canadian Perinatal Surveillance System. JAMA.2000;284:843-9.

18. Chen HF, Ho CA, Li CY. Increased risks of hip fracture in diabetic patients of Taiwan: A population-based study. Diabetes Care. 2008;31:75-80

19. Culter S, Ederer F. Maximum utilization of the life table method in analyzing survival. J Chronic Dis. 1958;53:457-81.

20. Vogel S, Huguelet P. Factors associated with multiple admissions to a public psychiatric hospital. Acta. Psychiatr. Scand.1997; 95:244-53.

21. Woo BK, Golshan S, Allen EC, Daly JW, Jeste DV, Sewell DD. Factors associated with frequent admissions to an acute geriatric psychiatric inpatient unit. J. Geriatr. Psychiatry Neurol. 2006;19:226-30.

22. Mahendran R, Mythily, Chong SA, Chan YH. Brief communication: factors affecting rehospitalisation in psychiatric patients in Singapore. Int. J. Soc. Psychiatry.2005;51, 101-5.

23. Oiesvold T, Saarento O, Sytema S, Vinding H, Göstas G, Lönnerberg $\mathrm{O}$, et al. Predictors for readmission risk of new patients: the Nordic Comparative Study on Sectorized Psychiatry. Acta. Psychiatr. Scand.2000;101:367-73.

24. Romansky JB, Lyons JS, Lehner RK, West CM. Factors related to psychiatric hospital readmission among children and adolescents in state custody. Psychiatr. Serv.2003;54: 356-62.

25. Pedersen J, Aarkrog T. A 10-year follow-up study of an adolescent psychiatric clientele and early predictors of readmission. Nord. J. Psychiatry.2001;55:11-6.

26. Gastal FL, Andreoli SB, Quintana MI, Almeida GM, Leite SO, McGrath J. Predicting the revolving door phenomenon among patients with schizophrenic, affective disorders and non-organic psychoses. Rev. Saude. Publica. 2000;34:280-5

27. Mortensen PB, Eaton AW. Predictors for readmission risk in schizophrenia. Psychol. Med.1994;24:223-32.

28. Kent S, Yellowlees P. Psychiatric and social reasons for frequent rehospitalization. Hosp. Community Psychiatry.1994;45:347-50.

29. Korkeila JA, Lehtinen V, Tuori T, Helenius H. Frequently hospitalised psychiatric patients: a study of predictive factors. Soc. Psychiatry Psychiatr. Epidemiol.1998;33: 528-34.

30. Hoffmann H. Age and other factors relevant to the rehospitalization of schizophrenic outpatients. Acta. Psychiatr. Scand.1994; 89:205-10.

31. Louis D, Taroni F, Melotti R, Rabinowitz C, Vizioli M, Fiorini M, et al. Increasing appropriateness of hospital admissions in the EmiliaRomagna region of Italy. J. Health Serv. Res. Policy.2008;13:202-8. 